Bull. Korean Math. Soc. 49 (2012), No. 1, pp. 175-195

http://dx.doi.org/10.4134/BKMS.2012.49.1.175

\title{
HERMITE INTERPOLATION USING PH CURVES WITH UNDETERMINED JUNCTION POINTS
}

\author{
Jae Hoon Kong, Seung Pil Jeong, and Gwang Il Kim
}

\begin{abstract}
Representing planar Pythagorean hodograph (PH) curves by the complex roots of their hodographs, we standardize Farouki's double cubic method to become the undetermined junction point (UJP) method, and then prove the generic existence of solutions for general $C^{1}$ Hermite interpolation problems. We also extend the UJP method to solve $C^{2}$ Hermite interpolation problems with multiple $\mathrm{PH}$ cubics, and also prove the generic existence of solutions which consist of triple $\mathrm{PH}$ cubics with $C^{1}$ junction points. Further generalizing the UJP method, we go on to solve $C^{2}$ Hermite interpolation problems using two $\mathrm{PH}$ quintics with a $C^{1}$ junction point, and we also show the possibility of applying the modified UJP method to $G^{2}\left[C^{1}\right]$ Hermite interpolation.
\end{abstract}

\section{Introduction}

Pythagorean hodograph $(\mathrm{PH})$ curves are polynomial curves with a polynomial speed function. They were first introduced by Farouki and Sakkalis [13]. PH curves have several advantages in computing geometric quantities such as arc-length, curvature and energy. In CAGD (computer aided geometric design) and CAD (computer aided design), they have been used for rational offsets, pipe and canal surfaces, CNC (computer numerical control) machining, interpolation of discrete data, and control of digital motion along curved paths $[4,11,15,9,10]$.

In general, $\mathrm{PH}$ curves can be completely characterized by expressions in the Bézier-Bernstein form [13,5] or by Clifford algebra [3]. In the plane, they can also be characterized by the complex roots of their hodographs [21]. There has been a lot of rigorous research on the use of each method of characterization, for both the planar $[28,31,32,12]$ and spatial cases $[14,7,19,24]$, as well as results for Minkowski PH (MPH) curves [2, 25, 29]. Early applications were

Received September 30, 2010; Revised July 20, 2011.

2010 Mathematics Subject Classification. Primary 68U05, 65D18, $68 \mathrm{U} 07$.

Key words and phrases. Pythagorean hodograph (PH) curve, complex representation, $C^{1}\left(C^{2}\right)$ Hermite interpolation, $G^{2}\left[C^{1}\right]$ Hermite interpolation, undetermined junction point (UJP) method.

This research was supported by the Korea Science and Engineering Foundation (KOSEF) grant funded by the Korea government(MEST) (2009-0073488). 
based on the representative expressions for $\mathrm{PH}$ curves, whereas more recent works have used tools specific to $\mathrm{PH}$ curves, such as $\mathrm{PH}-$ preserving mappings $[21,22]$ and speed reparameterization [23]. These applications have involved many Hermite interpolation problems: $G^{1}, C^{1}, G^{2}$ and $C^{2}$ interpolation in $\mathbb{R}^{2}$ $[11,32,12,23,1,18,8]$ and $\mathbb{R}^{3}[21,7,26] ; G^{1}$ and $C^{1}$ interpolation in the Minkowski spaces $\mathbb{R}^{1,1}, \mathbb{R}^{2,1}$ and $\mathbb{R}^{3,1}[2,29,20]$.

Some of these approaches have particular merits because of their algebraic computability and flexibility in applications. Farouki and Peters [12] solved $C^{1}$ Hermite interpolation problems using two consecutive $\mathrm{PH}$ cubics with a $G^{1}$ junction point. They compared $C^{1} \mathrm{PH}$ quintic interpolants with Hermite interpolants obtained by the same method after relaxing the $C^{1}$ Hermite condition at the junction point; but they provided no proof of the existence of solutions. Farouki and Albrecht [1] introduced an interesting method for constructing $C^{2}$ $\mathrm{PH}$ interpolating splines, which consist of several piece of $\mathrm{PH}$ quintics with $C^{2}$ junction points; however this approach goes through long complicate computational processes to solve a global nonlinear system of quadratic equations. Jüttler [18] introduced another method to solve $G^{2}\left[C^{1}\right]$ Hermite interpolation problems with $\mathrm{PH}$ septics, which requires relatively shorter computational processes than the previous one. He characterized the condition of a $\mathrm{PH}$ curve geometrically, and used numerical methods to explain the existence of solutions. There have also been several results on $C^{2} \mathrm{PH}$ curves $[30,27]$ and $G^{2}$ $\mathrm{PH}$ curves [33, 16, 17].

In this paper, we represent $\mathrm{PH}$ curves by the complex roots of their hodographs, and this representation plays a prominent part in the proofs of our main theorems. Using this representation, we standardize the double cubic method [12] and prove the existence of a solution for general $C^{1}$ Hermite interpolation problems. We call this method the undetermined junction point (UJP). We go on to extend this method, and show that, for $C^{2}$ Hermite interpolation problems, solutions given by triple $\mathrm{PH}$ cubics with two $C^{1}$ junction points exist generically. Moreover, by generalizing our extended method even further, we show that it can be applied to several geometric Hermite interpolation problems with diverse boundary conditions, such as $G^{2}$ Hermite interpolation and $G^{2}\left[C^{1}\right]$ Hermite interpolation [18], including $C^{2}$ interpolation. For example, we show that solutions of $G^{2}\left[C^{1}\right]$ Hermite interpolation problems, using $\mathrm{PH}$ curves with $\mathrm{PH}$ quintics and an undetermined junction point, exist generically with a curvature discontinuity at a single point. Moreover, we also show that, by manipulating the acceleration components of the boundary conditions, the curvature discontinuity at the junction point might be removed empirically and a smooth $G^{2}\left[C^{1}\right]$ interpolant can be obtained.

\section{Characterization of plane $\mathbf{P H}$ curves by hodograph roots}

In general, there are many ways of characterizing planar PH curves, using the Bézier-Berstein form, the complex roots of their hodographs, or Clifford algebra. In this paper, we use the complex representation introduced by Farouki 
[13], which is $\alpha(t)=u(t)+i v(t)$ instead of $\alpha(t)=(u(t), v(t))$. This representation allows us to regard PH curves as complex-valued polynomials. Moreover, we can restate the definition of $\mathrm{PH}$ curve and characterize $\mathrm{PH}$ curves using their hodograph roots as follows:

Definition 1. Let $\alpha(t)=u(t)+i v(t)$ be a plane curve, in which $u(t)$ and $v(t)$ are real polynomials. Then $\alpha(t)$ is called a Pythagorean-hodograph curve $(\mathrm{PH}$ curve) if and only if there exists a polynomial $\sigma(t)$ such that $\left\|\alpha^{\prime}(t)\right\|^{2}=\sigma(t)^{2}$; where || || signifies the complex norm.

Theorem 2.1 ([21]). A polynomial curve of degree $n$ is a Pythagorean hodograph curve if and only if its hodograph has only real roots and pairs of complex roots, of which one is equal or conjugate to the other.

Remark 2.2. A polynomial curve $\alpha(t)=u(t)+i v(t)$ is said to be regular if $\left\|\alpha^{\prime}(t)\right\| \neq 0$ for all $t$. Thus, $\alpha(t)$ is regular if $\alpha^{\prime}(t)$ has no real root. In addition, if $\alpha^{\prime}(t)$ has only non-real complex roots of even multiplicity, of which any two are not conjugate mutually, then the complex polynomial $\alpha^{\prime}(t)$ has no real factor polynomial (that is, $u^{\prime}(t)$ and $v^{\prime}(t)$ are relatively prime). In this case, we say that $\alpha(t)$ is regular if its hodograph has no real root, and strongly regular if the hodograph has only non-real complex roots of even multiplicity, of which any two are not mutually conjugate. For example, the general strongly regular $\mathrm{PH}$ cubics can be written as $\int k(t-c)^{2} d t$, where $c$ is a non-real complex number and $k$ is a constant complex number. The general strongly regular $\mathrm{PH}$ quintics are written $\int k\left(t-c_{1}\right)^{2}\left(t-c_{2}\right)^{2} d t$, where $c_{1}$ and $c_{2}$ are non-real complex numbers which are not mutually conjugate.

Note that, we deal with strongly regular PH curves throughout this paper.

\section{3. $C^{1}$ Hermite interpolation using $\mathrm{PH}$ cubic curves of type $\int k(t-c)^{2} d t$ with an undetermined $C^{1}$ junction point}

$\mathrm{PH}$ cubics have several merits in many practical applications for curve design, which stem from the nice properties of their geometric shape and their algebraic advantages in computation. So, there have been diverse researches using them $[32,12,29,26]$. A disadvantage of $\mathrm{PH}$ cubics is that they have only six degrees of freedom. This largely restricts their applicability to $G^{1}$ Hermite interpolation problems. We cannot use $\mathrm{PH}$ cubics to solve general $C^{1}$ Hermite interpolation problems, because $C^{1}$ Hermite data imposes more constraints than the degree of freedom of interpolants. We can use $\mathrm{PH}$ quintics which have eight degrees of freedom; but there have been some attempts to overcome this weak point $[12,23,1]$. In particular, Farouki and Peters [12] compared the effectiveness of double cubic interpolants and $\mathrm{PH}$ quintic interpolants in satisfying $C^{1}$ data. We standardize this method and provide the missing proof of the generic existence of a solution, together with a singularity analysis and some examples. 
We begin by noting that we will use the complex representation of a $\mathrm{PH}$ curve, so that all the elements of Hermite data will be complex numbers. For example, we use the complex numbers $P_{0}$ and $P_{1}$ to denote the starting and the ending points of interpolants, while their starting and terminal velocities are the complex numbers $V_{0}$ and $V_{1}$. Thus, a $C^{1}$ Hermite data $H_{C}^{1}$ is expressed as $H_{C}^{1}=$ $\left\{P_{0}, P_{1}, V_{0}, V_{1}\right\} . C^{2}$ Hermite data requires the additional complex numbers $A_{0}$ and $A_{1}$ to denote the accelerations of the interpolants at the end-points. Thus, a $C^{2}$ Hermite data $H_{C}^{2}$ can be expressed as $H_{C}^{2}=\left\{P_{0}, P_{1}, V_{0}, V_{1}, A_{0}, A_{1}\right\}$.

Definition 2. Let $\alpha:[0,1] \rightarrow \mathbb{R}^{2}$ and $\beta:[0,1] \rightarrow \mathbb{R}^{2}$ be two continuous plane curves. A point $Q$ is called the $C^{0}$ junction point of $\alpha(t)$ and $\beta(t)$ if $\alpha(1)=Q=\beta(0)$.

Remark 3.1. The precise type of a junction point of two curves is determined by the smoothness of the junction. For example, if the curves have the same velocity at the junction point, it is called a $C^{1}$ junction.

Theorem 3.2. For a given $C^{1}$ Hermite data $H_{C}^{1}=\left\{P_{0}, P_{1}, V_{0}, V_{1}\right\}$, there generically exist four interpolants, each of which consists of two PH cubics with a $C^{1}$ junction point.

Proof. Let $\alpha(t)$ and $\beta(t)$ be PH cubics. By Theorem 2.1 and Remark 2.2, they are given by $\alpha(t)=\frac{1}{3} k_{1}\left(t-c_{1}\right)^{3}+d_{1}$ and $\beta(t)=\frac{1}{3} k_{2}\left(t-c_{2}\right)^{3}+d_{2}$. Assume that $\alpha(t)$ and $\beta(t)$ comprise an interpolant for the Hermite data $H_{C}^{1}$ with a velocity vector $W$ and a $C^{1}$ junction point $Q$. Then, since we have

$$
\begin{gathered}
\alpha(0)=P_{0}, \alpha(1)=Q=\beta(0), \beta(1)=P_{1}, \\
\alpha^{\prime}(0)=V_{0}, \alpha^{\prime}(1)=W=\beta^{\prime}(0), \beta^{\prime}(1)=V_{1},
\end{gathered}
$$

we obtain

$$
\begin{aligned}
& P_{0}=-\frac{1}{3} k_{1} c_{1}^{3}+d_{1}, \quad P_{1}=\frac{1}{3} k_{2}\left(1-c_{2}\right)^{3}+d_{2}, \\
& Q=\frac{1}{3} k_{1}\left(1-c_{1}\right)^{3}+d_{1}, \quad Q=-\frac{1}{3} k_{2} c_{2}^{3}+d_{2}, \\
& V_{0}=k_{1} c_{1}^{2}, \quad V_{1}=k_{2}\left(1-c_{2}\right)^{2}, \\
& W=k_{1}\left(1-c_{1}\right)^{2}, \quad W=k_{2} c_{2}^{2} .
\end{aligned}
$$

Next, by eliminating $k_{1}$ and $k_{2}$ in (3) and (4), we obtain

$$
\begin{aligned}
& W=\left(\frac{c_{1}-1}{c_{1}}\right)^{2} V_{0}, \\
& W=\left(\frac{c_{2}}{c_{2}-1}\right)^{2} V_{1} .
\end{aligned}
$$

We eliminate $d$, from (1) and (2), and then use $k_{1}=\frac{V_{0}}{c_{1}^{2}}$, from (3), and obtain

$$
Q=\frac{V_{0}\left(1-3 c_{1}+3 c_{1}^{2}\right)}{3 c_{1}^{2}}+P_{0} .
$$


Similarly, using $k_{2}=\frac{V_{1}}{\left(1-c_{2}\right)^{2}}$, we can obtain

$$
Q=\frac{-V_{1}\left(1-3 c_{2}+3 c_{2}^{2}\right)}{3\left(c_{2}-1\right)^{2}}+P_{1}
$$

By substituting (3) into (1), we obtain

$$
\begin{aligned}
& d_{1}=\frac{1}{3} V_{0} c_{1}+P_{0}, \\
& d_{2}=\frac{1}{3} V_{1}\left(c_{2}-1\right)+P_{1} .
\end{aligned}
$$

Thus, by eliminating $W$ from (5) and (6), we have

$$
c_{2}=\frac{V_{*}\left(c_{1}-1\right)}{V_{*}\left(c_{1}-1\right)-c_{1}}, \quad \text { where } V_{*}= \pm \sqrt{\frac{V_{0}}{V_{1}}} .
$$

Finally, substituting (9) into (8), and eliminating $Q$ from (7) and (8), we get

$$
c_{1}=\frac{4 V_{0}}{5 V_{0}+V_{1} V_{*} \pm \sqrt{D}},
$$

where $D=-7 V_{0}^{2}+25 V_{0} V_{1}+10 V_{0} V_{1} V_{*}-24(P 0-P 1) V_{0}$. Also, by substituting (10) into (9), we obtain

$$
c_{2}=\frac{-4 V_{0}}{5 V_{0}+V_{0} V_{*} \pm V_{*} \sqrt{D}}+1 .
$$

From (9), (10) and (11), we can derive four possible pairs of $c_{1}$ and $c_{2}$, each of which generates one possible interpolant. This completes the proof.

Example 1. Consider a $C^{1}$ Hermite data $H_{C}^{1}$ given by $\{0,10,1+2 i, 1-i\}$. Let $\alpha(t)$ and $\beta(t)$ be $\mathrm{PH}$ cubics satisfying $\alpha(0)=0, \beta(1)=10, \alpha^{\prime}(0)=1+2 i$ and $\beta^{\prime}(1)=1-i$, with an undetermined $C^{1}$ junction point $Q$, so that $Q=$ $\alpha(1)=\beta(0)$ and $\alpha^{\prime}(1)=\beta^{\prime}(0)$. Then, from using (10) and (11), we can obtain four pairs of hodograph roots $c_{1_{j}}, c_{2_{j}}$ of the $\mathrm{PH}$ cubics as follows:

$$
\begin{array}{ll}
\left(c_{1_{1}}=-0.378471882-0.590246678 i,\right. & \left.c_{2_{1}}=1.479887368-0.290164571 i\right), \\
\left(c_{1_{2}}=-0.300968879-0.470234115 i,\right. & \left.c_{2_{2}}=0.761079611+0.053724378 i\right), \\
\left(c_{1_{3}}=0.240110218+0.104424060 i,\right. & \left.c_{2_{3}}=0.794270176+0.067086763 i\right), \\
\left(c_{1_{4}}=0.272617369+0.100007301 i,\right. & \left.c_{2_{4}}=1.342568704-0.260100907 i\right) .
\end{array}
$$

We can also obtain the junction points from (8):

$$
\begin{aligned}
& Q_{1}=5.120400906+0.157057522 i, \\
& Q_{2}=6.477112829+0.555724635 i, \\
& Q_{3}=4.879599093-0.407057522 i, \\
& Q_{4}=3.522887166+0.194275368 i,
\end{aligned}
$$

which correspond to the previous cases, in order. 
Thus, as shown in Figure 1-(a), we can get four interpolants, each of which consists of two $\mathrm{PH}$ cubics $\alpha_{j}$ and $\beta_{j}$ with the $C^{1}$ junction point $Q_{j}$ as follows:

$$
\begin{aligned}
\alpha_{1}(t)= & \left(t+3.171 t^{2}+0.949 t^{3}\right)+\left(2 t+0.339 t^{2}-1.182 t^{3}\right) i, \\
\beta_{1}(t)= & \left(5.120+10.190 t-6.742 t^{2}+1.431 t^{3}\right) \\
& +\left(1.157-0.868 t-0.735 t^{2}+0.446 t^{3}\right) i, \\
\alpha_{2}(t)= & \left(t+3.983 t^{2}+1.494 t^{3}\right)+\left(2 t+0.423 t^{2}-1.867 t^{3}\right) i, \\
\beta_{2}(t)= & \left(6.477+13.449 t-17.328 t^{2}+7.403 t^{3}\right) \\
& +\left(0.556-2.755 t+4.844 t^{2}-2.644 t^{3}\right) i, \\
\alpha_{3}(t)= & \left(t-6.549 t^{2}+10.428 t^{3}\right)+\left(2 t-5.481 t^{2}+3.074 t^{3}\right) i, \\
\beta_{3}(t)= & \left(4.880+19.187 t-24.014 t^{2}+9.947 t^{3}\right) \\
& +\left(-0.407+0.260 t+1.701 t^{2}-1.554 t^{3}\right) i, \\
\alpha_{4}(t)= & \left(t-5.605 t^{2}+8.128 t^{3}\right)+\left(2 t-5.280 t^{2}+3.474 t^{3}\right) i, \\
\beta_{4}(t)= & \left(3.523+14.174 t-9.916 t^{2}+2.220 t^{3}\right) \\
& +\left(0.194+1.863 t-3.309 t^{2}+1.251 t^{3}\right) i,
\end{aligned}
$$

where the component curves $\alpha_{j}$ and $\beta_{j}$ of each interpolant are determined by the values of the roots $\left(c_{1_{j}}\right.$ and $\left.c_{2_{j}}\right)$.

TABLE 1. Bending energy comparison of the UJP interpolants and the $\mathrm{PH}$ quintic interpolants for the $C^{1}$ Hermite data $\{0,10,1+2 i, 1-i\}$.

\begin{tabular}{|c||c|c|}
\hline$j$ & (a) UJP method & (b) PH quintic \\
\hline 1 & 0.822 & 2.419 \\
\hline 2 & 1289.217 & 3420.961 \\
\hline 3 & 643.161 & 453.832 \\
\hline 4 & 178.070 & 1563.548 \\
\hline
\end{tabular}

Moreover, comparing these four interpolants with the ones given by $\mathrm{PH}$ quintics [11] for the same $C^{1}$ Hermite data (see Figure 1-(b)), as shown Figure 1-(c) and in Table 1, we can obtain an UJP interpolant with better shape than the best $\mathrm{PH}$ quintic interpolant, in the sense that it has lower bending energy which is defined by $\int_{\gamma} \kappa^{2} d s$ where $\gamma$ is a plane curve and $\kappa$ is the curvature function of $\gamma$ (for details, see [6]).

Remark 3.3. In general, a $C^{1}$ Hermite interpolant has the parameter domain of length 1, for example, $[0,1]$. But all the interpolants in Example 1 have that of length 2 . This is caused by the process joining two consecutive interpolants. However, by the following technical modification with three simple steps, for a given $C^{1}$ Hermite data, we can automatically obtain the $\mathrm{PH}$ interpolants with 


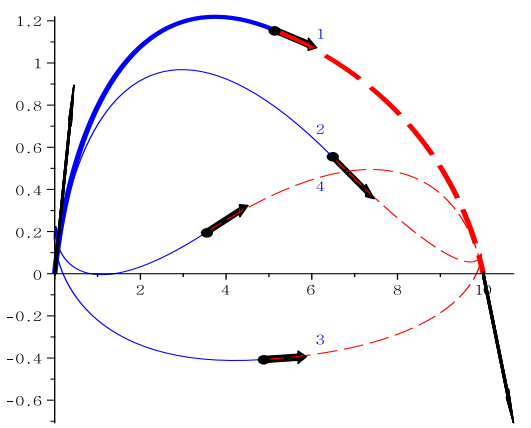

(a)

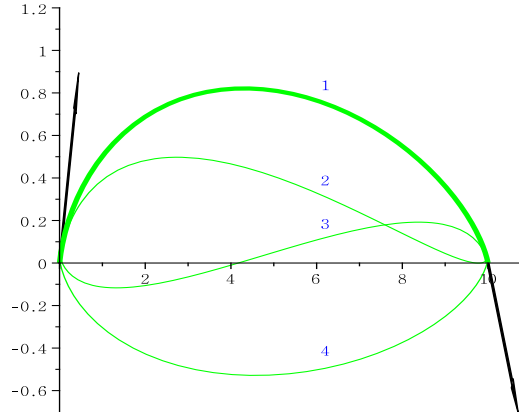

(b)

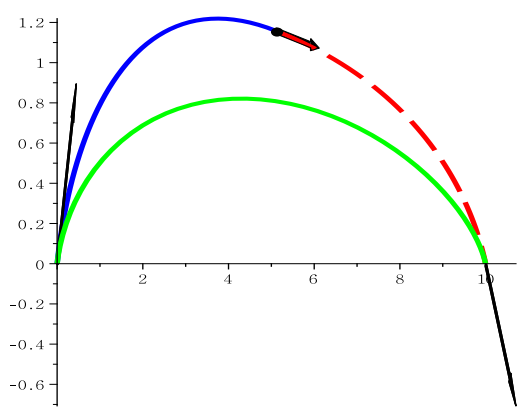

(c)

FiguRE 1. (a) shows four possible interpolants obtained by the UJP method for a $C^{1}$ Hermite data $\{0,10,1+2 i, 1-i\}$, and (b) shows four possible $\mathrm{PH}$ quintic interpolants for the same Hermite data. Each interpolant in (a) is annotated by a number of 1,2,3,4 assigned to the index $j$ in Example 1, and in (b) the annotating numbers are arbitrarily assigned. (c) shows both best interpolants respectively selected from (a) and (b), which their bending energies are compared in Table 1.

the general parameter domain: First, we modify the given $C^{1}$ Hermite data to a new $C^{1}$ Hermite data by reducing the size of tangent data by half. Next, using Theorem 3.2, we obtain the Hermite interpolants with two consecutive PH curves, as shown in Example 1, satisfying the new Hermite data. Finally, we replace the parameter $t$ of each piecewise interpolant with $2 t$ and reassume $t \in\left[0, \frac{1}{2}\right]$. For example, applying this modification to the Hermite data $H_{C}^{1}=$ $\{0,10,1+2 i, 1-i\}$ in Example 1, we can obtain four new Hermite interpolants with the general parameter domain, which are shown in Figure 2. In next examples, we do not mention especially about this modification any more, 
since the first and final steps of the modification are routine and automatical as you see. However, it is necessary to note that the change of the parameter domain might cause the change of the shape of interpolant (Compare Figure 1-(a) and Figure 2).

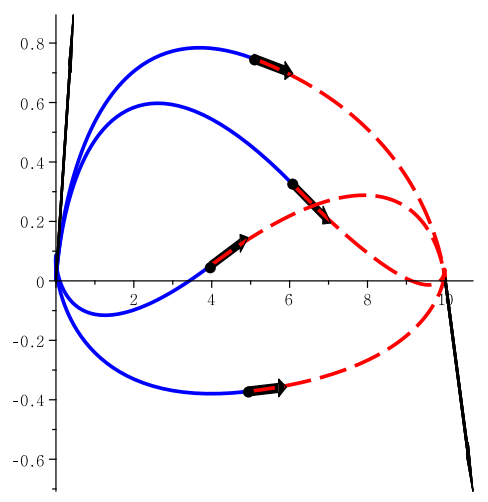

Figure 2. Four interpolants obtained by the technical modification introduced in Remark 3.3 for the $C^{1}$ Hermite data $H_{C}^{1}=\{0,10,1+2 i, 1-i\}$ in Example 1 .

Remark 3.4. As shown in Example 1, each $C^{1}$ UJP interpolant consists of two PH cubics. A strongly regular $\mathrm{PH}$ cubic should be a simple curve or a loop. Thus the $C^{1}$ UJP interpolant must be a combination of them. This means that, for a given $C^{1}$ Hermite data, there are four possible interpolants, and moreover that we can always obtain an interpolant which consists of two simple curves. This interpolant generically has the best shape in the UJP interpolants. Furthermore, by using the speed reparametrization method [23], we might obtain some $C^{1}$ UJP interpolants with bending energy as lower as possible.

Remark 3.5. Theorem 3.2 proves the generic existence of a solution using the UJP method. This leaves the possibility that the method may not work for some singular $C^{1}$ Hermite data, even though the probability of this situation occurring is zero. For example, consider the $C^{1}$ Hermite data $H_{C}^{1}=$ $\left\{P_{0}, P_{1}, V_{0}, V_{1}\right\}$, where $\frac{-4 V_{0}}{5 V_{0}+V_{0} V_{*} \pm V_{*} \sqrt{D}}+1=0, V_{*}= \pm \sqrt{\frac{V_{0}}{V_{1}}}$ and $D=-7 V_{0}^{2}+$ $25 V_{0} V_{1}+10 V_{0} V_{1} V_{*}-24(P 0-P 1) V_{0}$. Then from (6), (9) and (11) we have $c_{2}=0$, which is equivalent to $c_{1}=1$, and the UJP method does not work. However, we can deal with these unlikely irregular cases by speed reparametrization. This removes the singularity, and allows the UJP method to work normally. The details are explained in the following example.

Example 2. Let $H_{C}^{1}$ be a $C^{1}$ Hermite data given by $\{0,1,1+i, 2-i\}$, and let $\alpha(t)$ and $\beta(t)$ be PH cubics satisfying $\alpha(0)=0, \beta(1)=1, \alpha^{\prime}(0)=1+i$ 
and $\beta^{\prime}(1)=2-i$. In this case, four possible pairs of special hodograph roots $\left(c_{1}=1, c_{2}=0\right)$ exist, which implies that there is a singularity, so that the speed at the junction point is zero and the leading coefficients of the $\mathrm{PH}$ cubics diverge, so that the UJP method cannot work. However, by modifying $H_{C}^{1}$ to become $\{0,1,1+i, 0.25-0.125 i\}$, so that $\beta^{\prime}(1)=0.25-0.125 i$ instead of $\beta^{\prime}(1)=2-i$, we can simply remove the singularity. We can then obtain the following four pairs of non-singular hodograph roots $c_{1_{j}}, c_{2_{j}}$ :

$$
\begin{array}{ll}
\left(c_{1_{1}}=0.450171908+0.115872146 i,\right. & \left.c_{2_{1}}=0.732352065+0.032442086 i\right), \\
\left(c_{1_{2}}=0.519076683+0.114005772 i,\right. & \left.c_{2_{2}}=1.843351090-0.290767642 i\right), \\
\left(c_{1_{3}}=0.932131908-1.032326277 i,\right. & \left.c_{2_{3}}=0.626815215-0.041193814 i\right), \\
\left(c_{1_{4}}=1.144864477-0.779131387 i,\right. & \left.c_{2_{4}}=3.297481630+1.799519369 i\right) .
\end{array}
$$

Moreover, by applying the speed reparametrization method introduced by Kong et al [23]. We can finally obtain the four regular interpolants, shown in Figure 3 , which satisfy the original $C^{1}$ Hermite data $H_{C}^{1}$.

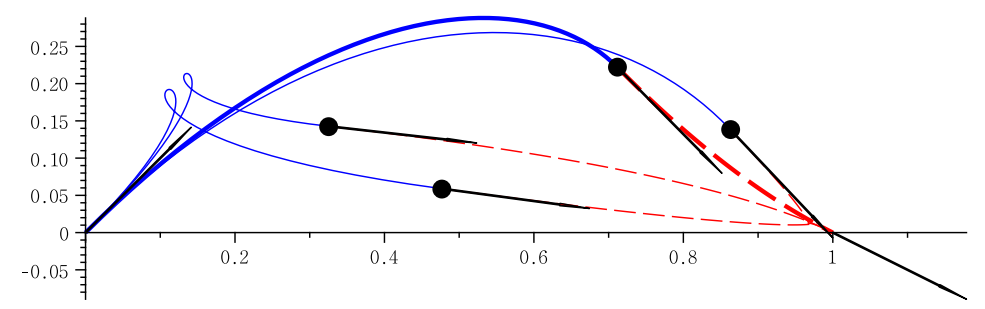

FIGURE 3. Four regular interpolants obtained by applying the speed reparametrization method to the $C^{1}$ Hermite data $\{0,1,1+i, 2-i\}$ in Example 2, by changing the final speed of $\beta$ from $2-i$ to $0.25-0.125 i$. The interpolants are determined by the hodograph roots $c_{1_{j}}$ and $c_{2_{j}}$ when $j=1,2,3$ and 4 respectively. The interpolant denoted by the thick lines is the interpolant with best shape, i.e., the lowest bending energy.

\section{4. $C^{2}$ Hermite interpolation using $\mathrm{PH}$ cubic curves of type $\int k(t-c)^{2} d t$ with three $C^{1}$ undetermined junction points}

The UJP method introduced in the previous section provides a new insight into solving Hermite interpolation problems. Previous method of solving these 
problem use a suitable single target curve with a moderate degree of freedom to match a given Hermite data. But the UJP method uses a special unit, consisting of multiple piecewise interpolants and their junction points, which satisfy specific constraints. For example, in the previous section, we used a unit made up of two piecewise $\mathrm{PH}$ cubics and one $C^{1}$ junction point.

This suggests a more general insight into tackling Hermite interpolation problems: If we use a special unit which consists of some specially designed piecewise interpolants and their junction points, there is hardly any need to handle curves of high degree, and we can find solutions consisting of piecewise curves of as low degree as possible, together with suitably designed junction points. In this section and the next, we present two extensive generalization of the UJP method.

First, we consider a modification of the UJP method to solve $C^{2}$ Hermite interpolation problems.

Theorem 4.1. For a $C^{2}$ Hermite data $H_{C}^{2}=\left\{P_{0}, P_{1}, V_{0}, V_{1}, A_{0}, A_{1}\right\}$, there generically exist four possible interpolants, each of which consists of four $P H$ cubics with their three $C^{1}$ junction points.

Proof. Let $\alpha(t)=k_{1}\left(t-c_{1}\right)^{3}+d_{1}, \beta(t)=k_{2}\left(t-c_{2}\right)^{3}+d_{2}, \gamma(t)=k_{3}\left(t-c_{3}\right)^{3}+d_{3}$ and $\delta(t)=k_{4}\left(t-c_{4}\right)^{3}+d_{4}$ be four $\mathrm{PH}$ cubic curves of type $\int k(t-c)^{2} d t$, which are joined in order at three $C^{1}$ junction points. We will denote these undetermined junction points as $Q_{j}(j=1,2,3)$ and the velocities at the junction points will be written $W_{j}(j=1,2,3)$. If this sequence of curves satisfies the $C^{2}$ Hermite data $H_{C}^{2}=\left\{P_{0}, P_{1}, V_{0}, V_{1}, A_{0}, A_{1}\right\}$, such that $\alpha(0)=$ $P_{0}, \alpha(1)=\beta(0)=Q_{1}, \beta(1)=\gamma(0)=Q_{2}, \alpha^{\prime}(0)=V_{0}, \alpha^{\prime}(1)=\beta^{\prime}(0)=W_{1}$, $\beta^{\prime}(1)=\gamma^{\prime}(0)=W_{2}, \gamma(1)=\delta(0)=Q_{3}, \delta(1)=P_{1}, \gamma^{\prime}(1)=\delta^{\prime}(0)=W_{3}$, $\delta^{\prime}(1)=V_{1}, \alpha^{\prime \prime}(0)=A_{0}$ and $\beta^{\prime \prime}(1)=A_{1}$. Then we have the followings:

$$
\begin{aligned}
& -k_{1} c_{1}^{3}+d_{1}=P_{0}, k_{1}\left(1-c_{1}\right)^{3}+d_{1}=-k_{2} c_{2}^{3}+d_{2}=Q_{1}, k_{1} c_{1}^{2}=V_{0}, \\
& k_{2}\left(1-c_{2}\right)^{3}+d_{2}=-k_{3} c_{3}^{3}+d_{3}=Q_{2}, k_{1}\left(1-c_{1}\right)^{2}=k_{2} c_{2}^{2}=W_{1}, \\
& k_{2}\left(1-c_{2}\right)^{2}=k_{3} c_{3}^{2}=W_{2}, k_{3}\left(1-c_{3}\right)^{3}+d_{3}=-k_{4} c_{4}^{3}+d_{4}=Q_{3}, \\
& k_{4}\left(1-c_{4}\right)^{3}+d_{4}=P_{1}, k_{3}\left(1-c_{3}\right)^{2}=k_{4} c_{4}^{2}=W_{3}, \\
& k_{4}\left(1-c_{4}\right)^{2}=V_{1},-2 k_{1} c_{1}=A_{0}, 2 k_{4}\left(1-c_{4}\right)=A_{1} .
\end{aligned}
$$

First, from (12), (13) and (14), we can obtain

$$
\begin{aligned}
& W_{1}=\left(\frac{1-c_{1}}{c_{1}}\right)^{2} V_{0}, W_{2}=\left(\frac{1-c_{2}}{c_{2}}\right)^{2} W_{1}, \\
& Q_{1}=\frac{\left(1-3 c_{1}+3 c_{1}^{2}\right) V_{0}}{3 c_{1}^{2}}+P_{0}, Q_{2}=\frac{\left(1-3 c_{2}+3 c_{2}^{2}\right) W_{1}}{3 c_{2}^{2}}+Q_{1},
\end{aligned}
$$

and from (14), (15) and (16), we also obtain

$$
W_{2}=\left(\frac{c_{3}}{1-c_{3}}\right)^{2} W_{3}, W_{3}=\left(\frac{c_{4}}{1-c_{4}}\right)^{2} V_{1}
$$




$$
Q_{3}=\frac{\left(1-3 c_{3}+3 c_{3}^{2}\right) W_{2}}{3 c_{3}^{2}}+Q_{2}, P_{1}=\frac{\left(1-3 c_{4}+3 c_{4}^{2}\right) W_{3}}{3 c_{4}^{2}}+Q_{3} .
$$

Next, from (12) and (16), we get

$$
c_{1}=-\frac{2 V_{0}}{A_{0}}, c_{4}=1-\frac{2 V_{1}}{A_{1}},
$$

and from (17) and (19), we also get

$$
\left(\frac{1-c_{1}}{c_{1}}\right)^{2}\left(\frac{1-c_{2}}{c_{2}}\right)^{2} V_{0}=\left(\frac{c_{3}}{1-c_{3}}\right)^{2}\left(\frac{c_{4}}{1-c_{4}}\right)^{2} V_{1} .
$$

Additionally, by using (18), (20) and (17), (19) in pairs, we get

$$
\begin{aligned}
& \frac{\left(1-3 c_{1}+3 c_{1}^{2}\right) V_{0}}{c_{1}^{2}}+\frac{\left(1-3 c_{2}+3 c_{2}^{2}\right) W_{1}}{c_{2}^{2}} \\
& +\frac{\left(1-3 c_{3}+3 c_{3}^{2}\right) W_{2}}{c_{3}^{2}}+\frac{\left(1-3 c_{4}+3 c_{4}^{2}\right) W_{3}}{c_{4}^{2}}=3\left(P_{1}-P_{0}\right),
\end{aligned}
$$

and $W_{1}=\left(\frac{1-c_{1}}{c_{1}}\right)^{2} V_{0}, W_{2}=\left(\frac{c_{3}}{1-c_{3}}\right)^{2}\left(\frac{c_{4}}{1-c_{4}}\right)^{2} V_{1}, W_{3}=\left(\frac{c_{4}}{1-c_{4}}\right)^{2} V_{1}$.

Let $\eta=\frac{1}{c_{2}}, \theta=\frac{1}{1-c_{3}}$. Then, from (21), (22) and (23), we get

(24) $\left(\frac{A_{0}}{2 V_{0}}+1\right)^{2} V_{0}(\eta-1)^{2}=\left(\frac{A_{1}}{2 V_{1}}-1\right)^{2} V_{1}(\theta-1)^{2}$,

(25) $\left(\frac{A_{0}}{2 V_{0}}+1\right)^{2} V_{0}\left(\eta^{2}-3 \eta+l_{1}\right)+\left(\frac{A_{1}}{2 V_{1}}-1\right)^{2} V_{1}\left(\theta^{2}-3 \theta+l_{2}\right)=3\left(P_{1}-P_{0}\right)$,

where $l_{1}=5+\frac{A_{0}}{2 V_{0}}, l_{2}=6+\frac{A_{1}\left(6 V_{1}-2 A_{1}\right)}{\left(A_{1}-2 V_{1}\right)^{2}}$. In addition, let $l_{3}=\left(\frac{A_{0}}{2 V_{0}}+1\right)^{2} V_{0}$, $l_{4}=\left(\frac{A_{1}}{2 V_{1}}-1\right)^{2} V_{1}$ and $l_{5}=3\left(P_{1}-P_{0}\right)$. Then from(24) and (25), we obtain two complex polynomials in the variables $\eta$ and $\theta$, as follows;

$$
\begin{aligned}
& l_{3}(\eta-1)^{2}=l_{4}(\theta-1)^{2}, \\
& l_{3}\left(\eta^{2}-3 \eta+l_{1}\right)+l_{4}\left(\theta^{2}-3 \theta+l_{2}\right)=l_{5} .
\end{aligned}
$$

We can rewrite (26) as $\eta=1 \pm \sqrt{\frac{l_{4}}{l_{3}}}(\theta-1)$ and, by substituting $\eta$ into (27), we finally obtain

$$
2 l_{4} \theta^{2}-\left(5 l_{4} \pm \sqrt{l_{3} l_{4}}\right) \theta+\left(l_{4}+l_{4} l_{2}+l_{3} l_{1}-2 l_{3} \pm \sqrt{l_{3} l_{4}}-l_{5}\right)=0 .
$$

Solving this equation over the complex number field $\mathbb{C}$, we generically obtain two complex roots. This completes the proof.

Example 3. Let $H_{C}^{2}$ be a $C^{2}$ Hermite data given by $H_{C}^{2}=\{1+i, 10+i, 2+$ $i, 1-2 i, 1+2 i, 2+3 i\}$, and let $\alpha(t)=k_{1}\left(t-c_{1}\right)^{3}+d_{1}, \beta(t)=k_{2}\left(t-c_{2}\right)^{3}+d_{2}$, $\gamma(t)=k_{3}\left(t-c_{3}\right)^{3}+d_{3}$ and $\delta(t)=k_{4}\left(t-c_{4}\right)^{3}+d_{4}$ be $\mathrm{PH}$ cubics which constitute an interpolant with three $C^{1}$ junction points $Q_{j}(j=1,2,3)$ that satisfy $H_{C}^{2}$ in the same order. Then, solving (28) for the Hermite data $H_{C}^{2}$, we get four possible values of $\theta_{j}(j=1,2,3,4)$, as follows;

$$
\theta_{1}=0.6783026555-1.478305562 i, \quad \theta_{2}=1.899534833+1.504361588 i,
$$


$\theta_{3}=0.6509730649-1.049792872 i, \quad \theta_{4}=1.771189827+1.023736913 i$.

TABLE 2. Four possible families of $C^{1}$ junction points.

\begin{tabular}{|c||c|c|c|}
\hline$j$ & $Q_{1_{j}}$ & $Q_{2_{j}}$ & $Q_{3_{j}}$ \\
\hline 1 & $3.467+3.183 i$ & $5.894+5.209 i$ & $10.483+4.467 i$ \\
\hline 2 & $3.467+3.183 i$ & $7.415+3.238 i$ & $10.483+4.467 i$ \\
\hline 3 & $3.467+3.183 i$ & $7.873+6.525 i$ & $10.483+4.467 i$ \\
\hline 4 & $3.467+3.183 i$ & $9.393+4.553 i$ & $10.483+4.467 i$ \\
\hline
\end{tabular}

TABLE 3. Four possible families of parameters which determine all the $\mathrm{PH}$ cubics from each interpolant.

\begin{tabular}{||c||c|c|c|c||}
\hline \multicolumn{1}{||c||}{$m$} & \multicolumn{4}{|c||}{1} \\
\hline \hline$j$ & 1 & 2 & 3 & 4 \\
\hline$k_{j_{m}}$ & $-0.033+0.183 i$ & $7.806+7.370 i$ & $-1.867+4.456 i$ & $-0.483+0.033 i$ \\
\hline$c_{j_{m}}$ & $-1.600+1.200 i$ & $0.376+0.024 i$ & $0.744-0.559 i$ & $1.615+1.077 i$ \\
\hline$d_{j_{m}}$ & $-0.467+1.267 i$ & $3.802+3.652 i$ & $9.780+5.342 i$ & $10.923+0.949 i$ \\
\hline \hline$m$ & \multicolumn{4}{|c||}{2} \\
\hline \hline$j$ & 1 & 2 & 3 & 4 \\
\hline$k_{j_{m}}$ & $-0.033+0.183 i$ & $11.828+3.935 i$ & $8.236-6.864 i$ & $-0.483+0.033 i$ \\
\hline$c_{j_{m}}$ & $-1.600+1.200 i$ & $0.336+0.098 i$ & $0.676+0.256 i$ & $1.615+1.077 i$ \\
\hline$d_{j_{m}}$ & $-0.467+1.267 i$ & $3.676+3.676 i$ & $11.166+4.786 i$ & $10.923+0.949 i$ \\
\hline \hline$m$ & \multicolumn{4}{|c||}{3} \\
\hline \hline$j$ & 1 & 2 & 3 & 4 \\
\hline$k_{j_{m}}$ & $-0.033+0.183 i$ & $0.080-0.125 i$ & $-1.678+2.224 i$ & $-0.483+0.033 i$ \\
\hline$c_{j_{m}}$ & $-1.600+1.200 i$ & $-1.881-2.599 i$ & $0.573-0.688 i$ & $1.615+1.077 i$ \\
\hline$d_{j_{m}}$ & $-0.467+1.267 i$ & $4.637+1.493 i$ & $11.228+4.958 i$ & $10.923+0.949 i$ \\
\hline \hline$m$ & \multicolumn{4}{||c||}{4} \\
\hline \hline$j$ & 1 & 2 & 3 & 4 \\
\hline \hline$k_{j_{m}}$ & $-0.033+0.183 i$ & $-0.105-0.838 i$ & $4.217-6.374 i$ & $-0.483+0.033 i$ \\
\hline$c_{j_{m}}$ & $-1.600+1.200 i$ & $-0.372-1.293 i$ & $0.577+0.245 i$ & $1.615+1.077 i$ \\
\hline$d_{j_{m}}$ & $-0.467+1.267 i$ & $4.723-1.555 i$ & $9.708+5.725 i$ & $10.923+0.949 i$ \\
\hline
\end{tabular}

We are now able to use the equations for $k_{j}, c_{j}, d_{j}$ and $Q_{j_{m}}(j=1,2,3,4$; $m=1,2,3)$ in the proof of Theorem 4.1 to obtain four interpolants, each of which consists of four PH cubics $\alpha_{j}, \beta_{j}, \gamma_{j}$ and $\delta_{j}$, with three $C^{1}$ junction points $Q_{j_{m}}(m=1,2,3)$ for each $j(j=1,2,3,4)$ as shown in Tables 2 and 3 .

Consequently, for the Hermite data $H_{C}^{2}$, we can obtain the four interpolants; one interpolant which consists of two simple curves and three interpolants which includes at least one loop, as shown in Figure 4.

Remark 4.2. As stated in Remark 3.5 and shown in Example 2, a regular interpolant may not exist for some $C^{2}$ Hermite data, even though the probability 


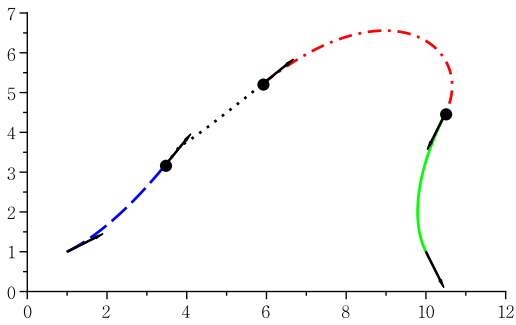

(a)

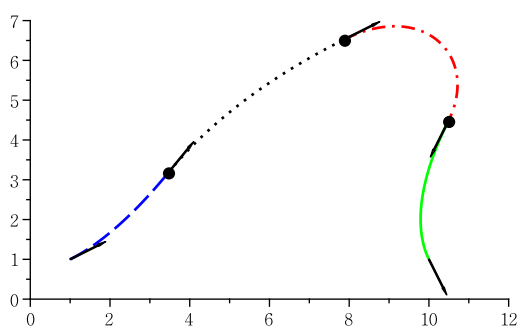

(c)

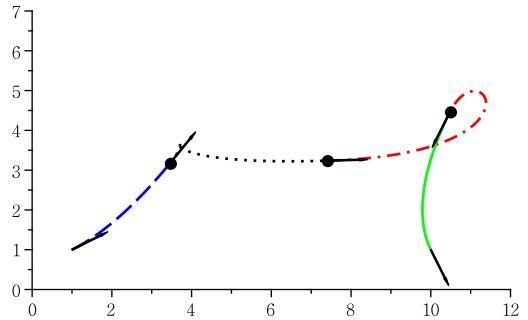

(b)

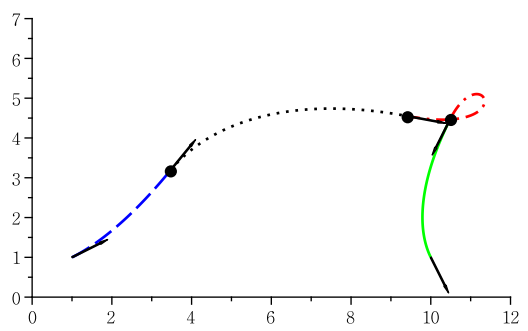

(d)

FIGURE 4. Four interpolants obtained by the UJP method using four PH cubics with three $C^{1}$ junction points for the $C^{2}$ Hermite data $H_{C}^{2}=\{1+i, 10+i, 2+i, 1-2 i, 1+2 i, 2+3 i\}$. Subfigures (a), (b), (c), (d) respectively show the interpolants determined by $\theta_{j}$ in Example 3 , when $j=1,2,3$ and 4 . The interpolant with best shape which consists of two simple curves is shown in (c).

of this is zero. But, in this case, we can again remove the singularity by speed reparametrization. 


\section{5. $C^{2}$ Hermite interpolation using $\mathrm{PH}$ quintics with an undetermined $C^{1}$ junction point}

The method introduced in the previous section, to solve $C^{2}$ Hermite interpolation problems using $\mathrm{PH}$ cubics with three $C^{1}$ junction points, seems to have two weaknesses: one is that it needs several $\mathrm{PH}$ cubics and junction points, and the other is that each of the $\mathrm{PH}$ cubic interpolants might have a curvature discontinuity at its junction points. We can improve the UJP method using $\mathrm{PH}$ cubics to address these issues by introducing a further modification of the UJP method for a $C^{2}$ Hermite data in which each interpolant is constructed from two $\mathrm{PH}$ quintics and a $C^{1}$ junction point. We can also apply this modified method to the $G^{2}\left[C^{1}\right]$ Hermite interpolation technique introduced by Jüttler [18].

First, we modify the UJP method to use two PH quintics with a $C^{1}$ junction point.

Theorem 5.1. For a $C^{2}$ Hermite data $H_{C}^{2}=\left\{P_{0}, P_{1}, V_{0}, V_{1}, A_{0}, A_{1}\right\}$, there generically exist four interpolants, each of which consists of two PH quintics with a $C^{1}$ junction point.

Proof. Let $H_{C}^{2}=\left\{P_{0}, P_{1}, V_{0}, V_{1}, A_{0}, A_{1}\right\}$ be a $C^{2}$ Hermite data and let $\alpha^{\prime}(t)=$ $\int_{0}^{t} k_{1}\left(\tau-c_{1}\right)^{2}\left(\tau-c_{2}\right)^{2} d \tau+d_{1}$ and $\beta^{\prime}(t)=\int_{0}^{t} k_{1}\left(\tau-c_{3}\right)^{2}\left(\tau-c_{4}\right)^{2} d \tau+d_{2}$ be $\mathrm{PH}$ quintics with a $C^{1}$ junction point $Q$, that satisfy $H_{C}^{2}$, in the same order.

Then, since $\alpha(0)=P_{0}, \alpha(1)=\beta(0)=Q, \beta(1)=P_{1}, \alpha^{\prime}(0)=V_{0}, \alpha^{\prime}(1)=$ $\beta^{\prime}(0)=W, \beta^{\prime}(1)=V_{1}, \alpha^{\prime \prime}(0)=A_{0}$ and $\beta^{\prime \prime}(1)=A_{1}$, we have

$$
d_{1}=P_{0} \text {, }
$$

$$
\begin{aligned}
& \frac{k_{1}}{30}\left(6-15 x_{1}+10 x_{1}^{2}+20 y_{1}-30 x_{1} y_{1}+30 y_{1}^{2}\right)+d_{1}=d_{2}=Q, \\
& \frac{k_{2}}{30}\left(6-15 x_{2}+10 x_{2}^{2}+20 y_{2}-30 x_{2} y_{2}+30 y_{2}^{2}\right)+d_{2}=P_{1}, \\
& k_{1} y_{1}^{2}=V_{0}, \\
& k_{1}\left(1-x_{1}+y_{1}\right)^{2}=k_{2} y_{2}^{2}, \\
& k_{2}\left(1-x_{2}+y_{2}\right)^{2}=V_{1}, \\
& -2 k_{1} x_{1} y_{1}=A_{0}, \\
& 2 k_{2}\left(1-x_{2}+y_{2}\right)\left(2-x_{2}\right)=A_{1},
\end{aligned}
$$

where $x_{1}=c_{1}+c_{2}, y_{1}=c_{1} c_{2}, x_{2}=c_{3}+c_{4}$ and $y_{2}=c_{3} c_{4}$.

From (31), (32) and (33), we can obtain

$$
\frac{V_{0}\left(1-x_{1}+y_{1}\right)^{2}}{y_{1}^{2}}=\frac{V_{1} y_{2}^{2}}{\left(1-x_{2}+y_{2}\right)^{2}},
$$

and from (31), (34) and (33), (35), we get

$$
y_{1}=-\frac{2 V_{0} x_{1}}{A_{0}},
$$




$$
y_{2}=\frac{4 V_{1}+\left(A_{1}-2 V_{1}\right) x_{2}-A_{1}}{A_{1}} .
$$

In addition, from $(29),(30),(31)$ and (32), we also obtain

$$
\frac{V_{1} f\left(x_{2}, y_{2}\right)}{30\left(1-x_{2}+y_{2}\right)^{2}}+\frac{V_{0} f\left(x_{1}, y_{1}\right)}{30 y_{1}^{2}}=P_{1}-P_{0},
$$

where $f(x, y)=6-15 x+10 x^{2}+20 y-30 x y+30 y^{2}$.

Next, using (37) and (38), we solve (36) for $x_{1}$, which yields

$$
x_{1}=\frac{a x_{2}+b}{c x_{2}+d},
$$

where $a=A_{0} V_{1} \sqrt{V_{0}}, b=-2 A_{0} V_{0} \sqrt{V_{1}}, c=A_{0} V_{1} \sqrt{V_{0}}-2 V_{0} V_{1} \sqrt{V_{1}}+2 V_{0} V_{1} \sqrt{V_{0}}+$ $A_{1} V_{0} \sqrt{V_{1}}$ and $d=-2 A_{0} V_{1} \sqrt{V 0}+4 V_{0} V_{1} \sqrt{V_{1}}-4 V_{0} V_{1} \sqrt{V_{0}}-A_{1} V_{0} \sqrt{V 1}$. We can then substitute (37) and (38) into (39). Combining this modified version of (39) with (40), we obtain a complex polynomial equation of degree 4 in $x_{2}$. By solving this equation, we can generically find four complex roots. This completes the proof.

Example 4. Let $H_{C}^{2}$ be the $C^{2}$ Hermite data given by $H_{C}^{2}=\{0,5,1+3 i, 2-$ $i, 1+2 i, 1+i\}$, and let $\alpha(t)=\int_{0}^{t} k_{1}\left(\tau-c_{1}\right)^{2}\left(\tau-c_{2}\right)^{2} d \tau+d_{1}$ and $\beta(t)=$ $\int_{0}^{t} k_{1}\left(\tau-c_{3}\right)^{2}\left(\tau-c_{4}\right)^{2} d \tau+d_{2}$ be, in the same order, $\mathrm{PH}$ quintics with a $C^{1}$ junction point satisfying $H_{C}^{2}$. Then, for $x_{1}=c_{1}+c_{2}, y_{1}=c_{1} c_{2}, x_{2}=c_{3}+c_{4}$ and $y_{2}=c_{3} c_{4}$, we have

$$
\begin{aligned}
& k_{1}=\frac{1+3 i}{y_{1}^{2}}, k_{2}=\frac{2-i}{\left(1-x_{2}+y_{2}\right)^{2}}, d_{1}=0, \\
& d_{2}=\frac{1+3 i}{30 y_{1}^{2}}\left(6-15 x_{1}+10 x_{1}^{2}+20 y_{1}-30 x_{1} y_{1}+30 y_{1}^{2}\right), \\
& y_{1}=-\frac{2(1+3 i) x_{1}}{1+2 i}, \\
& y_{2}=\frac{4(2-i)+(1+i-2(2-i)) x_{2}-1-i}{1+i} .
\end{aligned}
$$

Substituting (41) and (42) into (36) and (39), by solving them simultaneously, we obtain four pairs of solutions $x_{1_{j}}, x_{2_{j}}(j=1,2,3,4)$, as follows:

$$
\begin{array}{ll}
\left(x_{1_{1}}=0.0967498+0.0297784 i,\right. & \left.x_{2_{1}}=2.0323700+0.0814393 i\right), \\
\left(x_{1_{2}}=0.0384257-0.2473143 i,\right. & \left.x_{2_{2}}=2.0143658+0.1065499 i\right), \\
\left(x_{1_{3}}=0.1341668-0.2903794 i,\right. & \left.x_{2_{3}}=1.6683614-0.4090775 i\right), \\
\left(x_{1_{4}}=0.1184077+0.0256385 i,\right. & \left.x_{2_{4}}=1.8013613-0.1359923 i\right),
\end{array}
$$

from which we can also obtain four interpolants, each consisting of two $\mathrm{PH}$ quintics with a $C^{1}$ junction point that satisfies $H_{C}^{2}$, as shown in Figure 5.

Now, we will consider how to apply the modified UJP method introduced in this section to $G^{2}\left[C^{1}\right]$ Hermite interpolation problems. But, we first note 


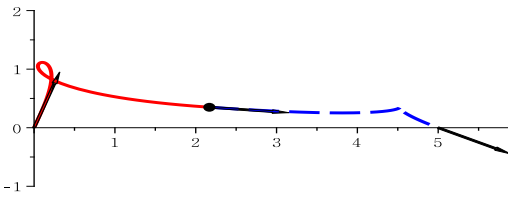

(a)

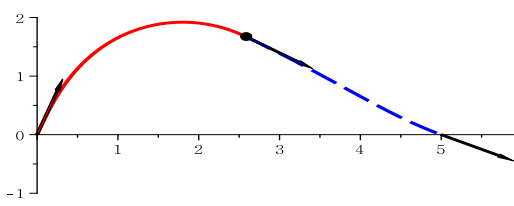

(c)

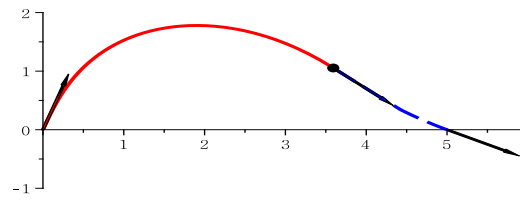

(b)

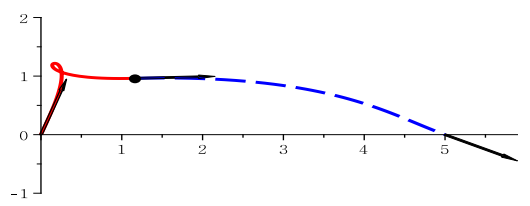

(d)

Figure 5. Four interpolants satisfying $H_{C}^{2}=\{0,5,1+3 i, 2-$ $i, 1+2 i, 1+i\}$, each consisting of two $\mathrm{PH}$ quintics and their $C^{1}$ junction point. Subfigures (a), (b), (c) and (d) show the interpolants determined by pairs of solutions $x_{1_{j}}$ and $x_{2_{j}}$ in Example 4 , when $j=1,2,3$ and 4 , respectively. The interpolant with best shape which consists of two simple $\mathrm{PH}$ quintics is shown in (c).

a potential drawback of this modified method, which is that the curvature function of each interpolant might have a discontinuity at its junction point. To proceed with this application, we therefore need another technique to eliminate this discontinuity. In the following example, we propose an empirical technique for this purpose.

Example 5. Consider a $G^{2}\left[C^{1}\right]$ Hermite data $H_{G^{2}\left[C^{1}\right]}=\left\{P_{0}=0, P_{1}=5, V_{0}=\right.$ $\left.1+3 i, V_{1}=2-i, \kappa_{0}=0.1, \kappa_{1}=-0.2\right\}$, where $\kappa_{0}$ and $\kappa_{1}$ respectively are the boundary curvatures of the target interpolants when $t=0,1$. Let us also consider another $C^{2}$ Hermite data $H_{C}^{2}=\left\{P_{0}=0, P_{1}=5, V_{0}=1+3 i, V_{1}=\right.$ $\left.2-i, A_{0}, A_{1}\right\}$, where $A_{0}=\lambda_{0} \frac{1+3 i}{\|1+3 i\|}+\sqrt{10} \kappa_{0} \frac{-3+i}{\|-3+i\|}$ and $A_{1}=\lambda_{1} \frac{2-i}{\|2-i\|}+$ $\sqrt{5} \kappa_{1} \frac{1-2 i}{\|1-2 i\|}$. Then, by Theorem 5.1, for each given $\lambda_{0}$ and $\lambda_{1}$, as shown in 
Figure 6 where $\lambda_{0}=0.5$ and $\lambda_{1}=1$, we can obtain four interpolants, each of which consists of two $\mathrm{PH}$ quintics with a $C^{1}$ junction point satisfying $H_{C}^{2}$. Let $\alpha(t)$ be an interpolant satisfying $H_{C}^{2}$ for each $\lambda_{0}$ and $\lambda_{1}$, and let $T$ and $N$ be the unit tangent vector field and the unit normal vector field on $\alpha(t)$. Then, since $\alpha^{\prime}(t)=v(t) T(t)$ for $v(t)=\left\|\alpha^{\prime}(t)\right\|$, we have $\alpha^{\prime \prime}(t)=v^{\prime}(t) T(t)+v(t) \frac{d T}{d t}=$ $v^{\prime}(t) T(t)+v(t)^{2} \kappa(t) N(t)$ by the Frenet theorem. This means that $\alpha(t)$ has the boundary curvatures $\kappa_{0}$ and $\kappa_{1}$ for any $\lambda_{0}$ and $\lambda_{1}$. This also implies that $\alpha(t)$ is an interpolant that will satisfy $H_{G^{2}\left[C^{1}\right]}$ for all $\lambda_{0}$ and $\lambda_{1}$.

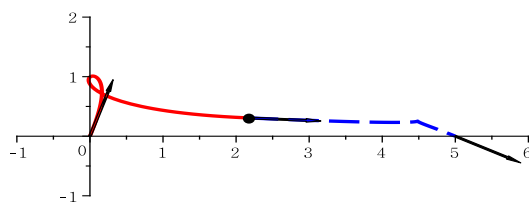

(a)

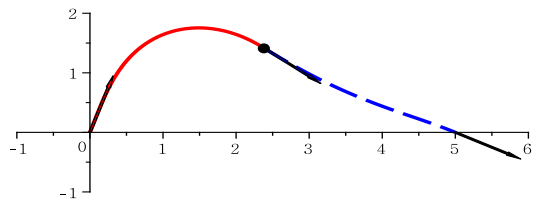

(c)

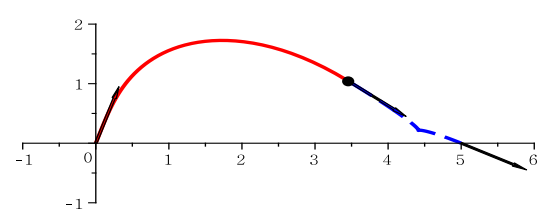

(b)

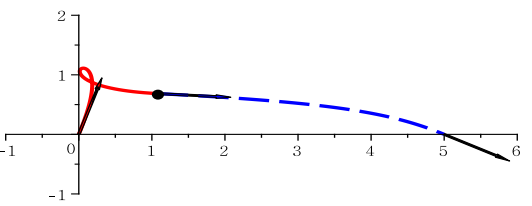

(d)

Figure 6. Four possible $G^{2}\left[C^{1}\right]$ interpolants obtained by the modified UJP method using two PH quintics, for a $G^{2}\left[C^{1}\right]$ Hermite data-set $H_{G^{2}\left[C^{1}\right]}=\left\{0,5 i, 1+3 i, 2-i, \kappa_{0}=0.1, \kappa_{1}=\right.$ $-0.2\}$, when $\lambda_{0}=0.5$ and $\lambda_{1}=1$. The interpolant with best shape is shown in (c).

However, in general, the curvatures of both the PH quintics of each interpolant satisfying $H_{C}^{2}$ are not equal at the junction point, as shown in Table 
4. This means that curvature continuity at the junction point is not always guaranteed.

TABLE 4. Comparison of the left curvature $\kappa_{\text {left }}$ and the right curvature $\kappa_{\text {right }}$ of each interpolant shown in Figure 6.

\begin{tabular}{||c||c|c||}
\hline & $\kappa_{\text {left }}$ & $\kappa_{\text {right }}$ \\
\hline (a) & 0.073 & 0.006 \\
\hline (b) & -0.205 & -0.037 \\
\hline (c) & -0.503 & 0.093 \\
\hline (d) & 0.180 & -0.015 \\
\hline
\end{tabular}

To remove the curvature discontinuity at the junction point, we propose an additional empirical technique. We first recall from the preceding paragraph that all interpolants satisfying a $C^{2}$ Hermite data $H_{C}^{2}$ must also satisfy the $G^{2}\left[C^{1}\right]$ Hermite data $H_{G^{2}\left[C^{1}\right]}=\left\{P_{0}, P_{1}, V_{0}, V_{1}, \kappa_{0}, \kappa_{1}\right\}$, if the acceleration vectors $A_{0}$ and $A_{1}$ are given, for parameters $\lambda_{0}$ and $\lambda_{1}$, as follows:

$$
A_{0}=\lambda_{0} T_{0}+v_{0} \kappa_{0} N_{0} \quad \text { and } \quad A_{1}=\lambda_{1} T_{1}+v_{1} \kappa_{1} N_{1}
$$

where $v_{j}=\left\|V_{j}\right\|, T_{j}=T(j)$ and $\left.N_{j}=N(j)\right)(j=0,1)$. So, by changing the parameters $\lambda_{0}$ and $\lambda_{1}$ (although in this example, we will only change the parameter $\lambda_{0}$ ) over $\mathbb{R}$, and by applying the modified UJP method to each $C^{2}$ Hermite data $H_{C}^{2}$, we can change the curvatures of the two $\mathrm{PH}$ quintics of each interpolant at the junction point, while continuing to satisfy $H_{G^{2}\left[C^{1}\right]}$. We repeat this procedure until the curvatures of the two $\mathrm{PH}$ quintics comprising the interpolant are equal at the junction point. All being well, we can now at last obtain the interpolant without a curvature discontinuity at the junction point.

As an exercise, we will apply this technique to the interpolant shown in Figure 6-(c). Figure 7-(a) and (b) show that the curvature inequality between the two PH quintics of the interpolant can be reversed. This means that the technique works, equivalently, that there exists a $G^{2}\left[C^{1}\right]$ interpolant satisfying $H_{G^{2}\left[C^{1}\right]}$ with curvature continuity at the junction point. When $\lambda_{0}=-12.989$, we can approximate the $G^{2}\left[C^{1}\right]$ Hermite interpolant without the curvature discontinuity numerically, and we see that it satisfies $H_{G^{2}\left[C^{1}\right]}$, as shown in Figure 7-(c).

\section{Concluding remarks and suggestions for further study}

We have introduced Hermite interpolation techniques that use planar $\mathrm{PH}$ curves represented by the complex roots of their hodographs. First, we standardized the double cubic method introduced by Farouki to become the new UJP method, and gave a proof of the generic existence of solutions to general $C^{1}$ Hermite interpolation problems. In addition, we extended the UJP method 


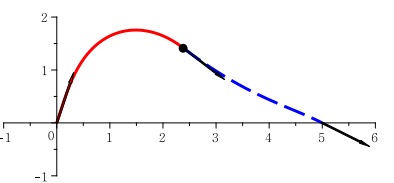

(a)

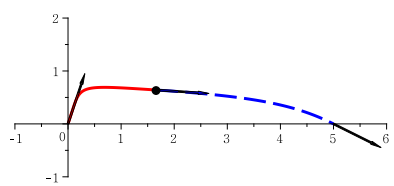

(b)

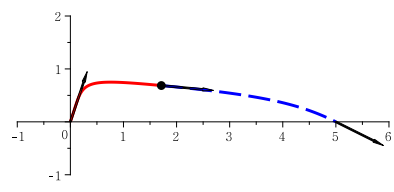

(c)

FiguRE 7. Reversal of the curvature inequality is observed only as the parameter $\lambda_{0}$ is varied from 0.5 to -14 , while keeping $\lambda_{1}$ at 1 , with an interpolant constructed from two $\mathrm{PH}$ quintics of the same type, as shown in Figure 6-(c), and an interpolant determined numerically with a sufficiently small curvature difference at the junction point: (a) shows the interpolant with $\kappa_{\text {left }}=-0.503, \kappa_{\text {right }}=0.093$ when $\lambda_{0}=0.5$; (b) shows the interpolant with $\kappa_{\text {left }}=0.001, \kappa_{\text {right }}=-0.022$ when $\lambda_{0}=-14$; and (c) shows the interpolant with $\kappa_{\text {left }}=$ $-0.018180, \kappa_{\text {right }}=-0.018183$ when $\lambda_{0}=-12.989$.

to solve $C^{2}$ Hermite interpolation problems with multiple $\mathrm{PH}$ cubics and also proved the generic existence of a solution which consists of triple $\mathrm{PH}$ cubics with $C^{1}$ junction points. Also, by further generalization of the UJP method, we solved $C^{2}$ Hermite interpolation problems using two $\mathrm{PH}$ quintics with a $C^{1}$ junction point. In addition, we showed the possibility of applying the modified method to $G^{2}\left[C^{1}\right]$ Hermite interpolation problems.

We can suggest directions for further study. First is the shape analysis of the interpolants obtained by the UJP method, and related topics such as a complete theoretical understanding of the curvature continuity of interpolants 
at the junction point by a consideration of the $T_{j}$ components of the boundary accelerations, in the contest of the numerical technique introduced in Example 5. Second, we suggest further applications of the UJP method to other Hermite interpolation problems such as those involving asymmetric data. We are currently tackling some of these issues.

\section{References}

[1] G. Albrecht and R. T. Farouki, Construction of $C^{2}$ Pythagorean-hodograph interpolating splines by the homotopy method, Adv. Comput. Math. 5 (1996), no. 4, 417-442.

[2] H. I. Choi, C. Y. Han, H. P. Moon, K. H. Roh, and N. S. Wee, Medial axis transformation and offset curves by Minkowski Pythagorean hodograph curves, Computer-Aided Design 31 (1999), 59-72.

[3] H. I. Choi, D. S. Lee, and H. P. Moon, Clifford algebra, spin representation, and rational parametrization of curves and surfaces, Advances in Computational Mathematics 17 (2001), 5-48.

[4] R. T. Farouki, Pythagorean hodograph curves in practical use, Geometry processing for design and manufacturing, 3-33, SIAM, Philadelphia, PA, 1992.

[5] - The conformal map $z \rightarrow z^{2}$ of the hodograph plane, Comput. Aided Geom. Design 11 (1994), no. 4, 363-390.

[6] _ The elastic bending energy of Pythagorean-hodograph curves, Comput. Aided Geom. Design 13 (1996), no. 3, 227-241.

[7] R. T. Farouki, M. Al-Kandari, and T. Sakkalis, Hermite interpolation by rotationinvariant spatial Pythagorean-hodograph curves, Adv. Comput. Math. 17 (2002), no. 4, 369-383.

[8] R. T. Farouki and C. Y. Han, Rational approximation schemes for rotation-minimizing frames on Pythagorean-hodograph curves, Comput. Aided Geom. Design 20 (2003), no. 7, 435-454.

[9] R. T. Farouki, J. Manjunathaiah, D. Nicholas, G.-F. Yuan, and S. Jee, Variable-feedrate CNC interpolators for constant material removal rates along Pythagorean-hodograph curves, Computer Aided Geometric Design 3 (1998), 631-640.

[10] R. T. Farouki, J. Manjunathaiah, and G.-F. Yuan, G codes for the specification of Pythagorean-hodograph tool paths and associated feedrate functions on open-architecture CNC machines, International Journal of Machine Tools and Manufacture 39 (1999), 123-142.

[11] R. T. Farouki and C. A. Neff, Hermite interpolation by Pythagorean-hodograph quintics, Math. Comp. 64 (1995), no. 212, 1589-1609.

[12] R. T. Farouki and J. Peters, Smooth curve design with double-Tschirnhausen cubics, Ann. Numer. Math. 3 (1996), no. 1-4, 63-82.

[13] R. T. Farouki and T. Sakkalis, Pythagorean hodographs, IBM J. Res. Develop. 34 (1990), no. $5,736-752$.

[14] , Pythagorean hodograph space curves, Adv. Comput. Math. 2 (1994), no. 1, $41-66$.

[15] R. T. Farouki and S. Shah, Real-time CNC interpolator for Pythagorean hodograph curves, Comput. Aided Geom. Design 13 (1996), 583-600.

[16] Z. Habib and M. Sakai, $G^{2}$ Pythagorean hodograph quintic transition between two circles with shape control, Comput. Aided Geom. Design 24 (2007), no. 5, 252-266.

[17] _ Transition between concentric or tangent circles with a single segment of $G^{2}$ PH quintic curve, Comput. Aided Geom. Design 25 (2008), no. 4-5, 247-257.

[18] B. Jüttler, Hermite interpolation by Pythagorean hodograph curves of degree seven, Math. Comp. 70 (2001), no. 235, 1089-1111. 
[19] B. Jüttler and C. Mäurer, Cubic Pythagorean hodograph spline curves and applications to sweep surface modeling, Computer-Aided Design 31 (1999), 73-83.

[20] G. I. Kim and M. H. Ahn, $C^{1}$ Hermite interpolation using MPH quartic, Comput. Aided Geom. Design 20 (2003), no. 7, 469-492.

[21] G. I. Kim, J. H. Kong, and S. Lee, First order Hermite interpolation with spherical Pythagorean-hodograph curves, J. Appl. Math. Comput. 23 (2007), no. 1-2, 73-86.

[22] G. I. Kim and S. Lee, Pythagorean-hodograph preserving mappings, J. Comput. Appl. Math. 216 (2008), no. 1, 217-226.

[23] J. H. Kong, S. P. Jeong, S. Lee, and G. I. Kim, $C^{1}$ Hermite interpolation with simple planar PH curves by speed reparametrization, Comput. Aided Geom. Design 25 (2008), no. 4-5, 214-229.

[24] C. Manni, A. Sestini, R. T. Farouki, and C. Y. Han, Characterization and construction of helical polynomial space curves, J. Comput. Appl. Math. 162 (2004), no. 2, 365-392.

[25] H. P. Moon, Minkowsi Pythagorean hodographs, Comput. Aided Geom. Design 25 (2008), no. 4-5, 739-753.

[26] F. Pelosi, R. T. Farouki, C. Manni, and A. Sestini, Geometric Hermite interpolation by spatial Pythagorean hodograph cubics, Adv. Comput. Math. 22 (2005), no. 4, 325-352.

[27] F. Pelosi, M. L. Sampoli, R. T. Farouki, and C. Manni, A control polygon scheme for design of planar $C^{2}$ PH quintic spline curves, Comput. Aided Geom. Design 24 (2007), no. $1,28-52$.

[28] H. Pottmann, Curve design with rational Pythagorean-hodograph curves, Adv. Comput. Math. 3 (1995), no. 1-2, 147-170.

[29] Z. Šir and B. Jüttler, Euclidean and Minkowski Pythagorean hodograph curves over planar cubics, Comput. Aided Geom. Design 22 (2005), no. 8, 753-770.

[30],$C^{2}$ Hermite interpolation by Pythagorean hodograph space curves, Mathematics of Computation 76 (2007), no. 259, 1373-1391.

[31] D. J. Walton and D. S. Meek, A Pythagorean hodograph quintic spiral, Computer-Aided Design 28 (1996), no. 12, 943-950.

[32] _ Geometric Hermite interpolation with Tschirnhausen cubics, J. Comput. Appl. Math. 81 (1997), no. 2, 299-309.

[33] D. J. Walton and D. S. Meek, $G^{2}$ curve design with a pair of Pythagorean Hodograph quintic spiral segments, Comput. Aided Geom. Design 24 (2007), no. 5, 267-285.

\section{JAE HOON KONG}

Department of Mathematics

GyeOngSang National University

JinJu 660-701, KorEA

E-mail address: jhkong@gnu.ac.kr

Seung Pil JeOng

Department of Mathematics

GyeongSang National University

JinJu 660-701, KoreA

E-mail address: sumgjal@naver.com

GWANG IL KIM

Department of Mathematics and RINS

College of Natural Science

GyeongSang National University

JINJU 660-701, KOREA

E-mail address: gikim@gnu.ac.kr 